\title{
Treatment Challenges, Priorities, and Relationship with Healthcare Providers in HIV Care: A Cross-Sectional Survey of Portuguese Adults Living with HIV
}

\author{
Antonio Antunes MSc ${ }^{a}$, Ines Augusto PharmD ${ }^{b}$, Patricia Parada $\mathrm{MD}^{\mathrm{c}}$, Chinyere Okoli \\ PharmD $^{d}$, Ama Appiah MSc PharmD ${ }^{\mathrm{e}}$ and Patricia de los Rios MSc ${ }^{\dagger}$ \\ a Scientific Manager, ViiV Healthcare, Lisbon, Portugal \\ b Medical Science Liaison, ViiV Healthcare, Lisbon, Portugal \\ c Country Medical Lead, ViiV Healthcare, Lisbon, Portugal \\ d Global Scientific Advisor at ViiV Healthcare, Brentford, London UK \\ e European Scientific Advisor ViiV Healthcare, Brentford, London UK \\ f Global Medical Sciences Advisor at ViiV Healthcare, RTP, NC, USA
}

\begin{abstract}
Background: We focus on the World Health Organization's definition of health as it pertains to health-related quality of life, not just the "absence of disease or infirmity". We investigated various treatment challenges among People Living with HIV (PLHIV) in Portugal.

Methods: We analyzed data for 60 Portuguese adult PLHIV with a confirmed HIV diagnosis and on antiretroviral therapy (ART) who participated in the 2019 Positive Perspectives Survey. Data were summarized using percentages and compared with $\chi^{2}$ tests.

Results: Most participants reported they were virally suppressed (97\% [58/60]), men (78\% [47/60]), <50 years (73\% [44/60]), and with $\geq 1$ non-HIV comorbidity (70\% [42/60]). Overall, 15\% [9/60] reported difficulty ingesting medication; 35\% [21/60] experienced ART side effects; 22\% [13/60] felt daily oral ART limited their day-to-day life; 25\% [15/60] were stressed by their dosing schedule; 33\% [20/60] said daily oral dosing precipitated bad memories; while 63\% [38/60] felt daily dosing reminded them of their HIV status. These challenges were associated with treatment-avoidance behaviors; PLHIV reported missing ART $\geq 1$ time within the past month because of food requirements (27\% [16/60]); side effects (12\% [7/60]); concerns about long-term effects of ART (10\% [6/60]) and problems swallowing medication (5\% [3/60]). Overall, 88\% [53/60] were optimistic that future advances in HIV care would improve their overall wellbeing. Only 35\% [21/60] perceived no communication barriers with their providers; these individuals reported higher prevalence of optimal physical (86\% [18/21] vs. 49\% [19/39], $\mathrm{p}=0.005)$ and mental health $(86 \%$ [18/21] vs. 36\% [14/39], $\mathrm{p}<0.001)$ than those with a perceived barrier.

Conclusion: For some PLHIV, adhering to daily HIV regimens was linked with diverse emotional challenges, including pill fatigue and anxiety. Clinicians should consider patient preferences when prescribing ART and engaging PLHIV in treatment decisions.
\end{abstract}

\section{Keywords}

Clinical outcomes, emotional correlates, HIV, HIV medications, patient-physician communication, person-centered healthcare, PLHIV, Portugal, treatment adherence, unmet needs

\section{Correspondence address}

Mr. Antonio Antunes, ViiV Healthcare, Arquiparque-Miraflores, 1499-013 Algés, Lisbon, Portugal.

E-mail: antonio.x.antunes@viivhealthcare.com

Accepted for publication: 7 August 2020

\section{Introduction}

Portugal has made substantial progress towards the three "90" targets developed by the Joint United Nations Programme on HIV/AIDS (UNAIDS) to increase diagnosis among all people living with HIV (PLHIV), increase ART coverage among those diagnosed, and increase virologic suppression among those on treatment [1]. Little is known, however, about the fourth "90" (improved quality of life) among people living with HIV (PLHIV) in Portugal. A better understanding of the intersection between HIV and emotional, mental, and physical aspects of life, can inform holistic care. The aim of this study was to quantify physical, mental, and 
psychological challenges faced by PLHIV in Portugal; to explore how these challenges were associated with treatment avoidance behaviors and to determine the extent of patient-HCP engagement in addressing those challenges.

Treatment concerns among PLHIV may be distinct in many respects from those of other patients. HIV requires lifelong treatment on account of having no cure; there is also the added negative social dimension which can complicate acceptance of the disease, constrict social support, decrease the extent of adherence to treatment, and impair overall quality of life [2]. HIV illness, in general terms, presents multidimensional issues such as psychological trauma, HIV-Associated Neurocognitive Disorders and other neuropsychological disorders, negative feelings, stigma, relationship problems, and emotional stress [3-8]. Stigma directed at PLHIV is often multilayered, including self/internalized stigma, person-toperson discrimination, or structural/institutional discrimination (i.e., discriminatory policies) [9-11]. The immense physical, emotional, and psychological challenge associated with having to ingest HIV medication daily, cannot be underestimated [12].

A better understanding of how these issues collectively influence treatment-related perceptions and behaviors among PLHIV can help inform clinical practice, including patient counseling and treatment planning. Such consideration of treatment challenges is necessary even for PLHIV who currently have optimal adherence to treatment or are virologically suppressed because neither adherence nor viral suppression are definitive states, they are strongly intertwined with quality of life [13-16]. Indeed, quality of life, for better or for worse, could be both an effect, and a determinant, of treatment adherence. As an effect of treatment adherence, PLHIV may enjoy the benefits of being virally undetectable, but may suffer from some side effects of treatment. The role of quality of life as a determinant of treatment adherence is reflected from the observation that some of the top reasons for suboptimal adherence to HIV treatment are non-medical, rather than medical reasons [15]. Recognizing the strong link between patient-centered care and clinical outcomes, the fourth "90" target (improved quality of life for $\geq 90 \%$ of PLHIV) was proposed as a complement to the set of original three "90" targets [16,17]. Similarly, the World Health Organization's definition of health goes beyond merely ensuring "absence of disease or infirmity"; rather, it focuses on promoting health-related quality of life in all domains [18].

The patient-healthcare provider (HCP) relationship, including HIV care, is sometimes beset by unfulfilled expectations regarding the degree of engagement HCPs want from patients and vice versa [19-22]. HCPs encourage patients to adhere to treatment and keep their appointments [21]; yet various medical, psychological, or emotional challenges - some of which may be unknown to HCPs - may sabotage patients' adherence to their treatment and could lead to non-retention in care [23, 24]. At the same time, some PLHIV may need assistance from their HCPs to address personal treatment challenges [22]; paradoxically though, they may be hesitant sharing those concerns with their HCPs [25]. With reducing frequency of patient visits and high patient-provider ratios in certain regions [26,27], it is reasonable to expect that HCPs may not remember every detail from past patient encounters. Patients might also see different HCPs on successive consultations. A more proactive and person-centered approach is therefore needed to ensure a continuum of high-quality care. Patients need to be empowered to initiate discussions about treatment concerns rather than waiting for their HCPs to address the issue. Intensified efforts are also needed among HCPs to elicit any concern patients may have regarding their treatment.

The second wave of the Positive Perspectives Study was conducted in 25 countries to explore issues related to patient unmet needs, medication challenges, stigma, and treatment aspirations. Previous research conducted with pooled data from all 25 countries examined the single effects of various emotional, physical, and psychosocial challenges on health-related outcomes [24]. This study builds on the past work by further examining the joint effect of treatment challenges. We focus on Portugal in this paper because, despite remarkable strides made in HIV prevention and control in the country, Portugal still has the highest HIV incidence rate of all Western European countries, at 9.5 new diagnoses per 100,000 population during 2018 [28]. A better understanding of current treatment challenges as well as gaps in patient-provider communication can help HCPs to take proactive steps in managing newly diagnosed and treatment-experienced patients alike.

\section{Methods}

\section{Survey administration}

During 2019, a standardized, web-based, survey was conducted in 25 countries, including Portugal. This study, entitled, Positive Perspectives, had its inclusion criteria as PLHIV aged 18 years or older and on ART. In Portugal, the survey was conducted over two months, and ended on July $27^{\text {th }}$, 2019. A total of 159 individuals were screened, and of these, 60 met the inclusion criteria and completed the survey. Of the 60 participants, most were from Lisbon and Tagus Valley (68\%) and the remainder from Algarve, Center, Islands (Azores, Madeira), Porto and North. Five patient organizations assisted with recruitment and received honoraria for their collaboration, in full competence with legal requirements. All participants provided informed consent. This study was deemed as exempt research by Pearl-IRB (\#18-080622).

\section{Measures}

\section{Attitudes and behaviors towards HIV treatment}

Assessed constructs included perception that daily ART dosing: limits day-to-day life; is a daily self-reminder of HIV status; cues bad memories and increases the chances of others knowing their HIV status. Data were also collected on various concerns about HIV medications, 
including how taking ART for many years might impact their body and general health status; potential ART drugdrug interactions; missing ART doses as prescribed; running out of treatment options; and possibly having to take more and more medications with increasing age. Openness to trying innovative treatments was also assessed. Suboptimal adherence was defined as a report of $\geq 1$ reason for missing ART for $\geq 5$ times within the past month. We further assessed whether participants felt there was room for improvement with either their HIV medication, or their overall HIV management. The perception that there was room for improving current HIV medication was defined as a score of $\geq 4$ (on an ordinal scale from 1 to 5) in response to the question "Do you feel that there is room for improvement with your current HIV medication or do you feel that it fully meets your needs?” The perception that there was room for improving overall HIV management was defined as a response of "Agree” or "Strongly agree" to the statement "I feel there is room for improving the way my HIV is managed”.

\section{HCP and PLHIV engagement in care}

To measure HCP engagement in care, survey participants provided responses on the following indicators (1) "I am given enough information to be involved in making choices about my HIV treatment"; (2) "My provider seeks my views about treatment before prescribing an HIV medication"; (3) "My provider asks me if I have any concerns about the HIV medication I am currently taking"; (4) "My provider asks me frequently about any side effects I might be experiencing with my HIV treatment"; (5) "My provider tells me about new HIV treatment options that become available" and (6) "My provider has told me about 'undetectable = untransmittable' $(\mathrm{U}=\mathrm{U})$ ”.

To measure PLHIV (i.e., self) engagement in care, survey participants provided on the following indicators: (1) "I would like to be more involved when it comes to decisions about my HIV treatment"; (2) "I feel I understand enough about my HIV treatment"; (3) My "main HIV care provider meets [my] personal needs and takes into account the things that are most important to [me]"; (4) "satisfied ... with current HIV medication"; (5) "future advances in HIV treatment will improve my overall health and wellbeing" and (6) "My HIV medication prevents me from passing on HIV to others".

To measure the extent of both HCP and PLHIV engagement in care (low, moderate, high), we created a composite variable which was partially adapted from the Observing Patient Involvement scale, as previously reported [25,29]. The scale comprises multiple items across domains such as HCP facilitation of patient involvement, level of information exchange, and patient participation in the decision-making process.

\section{Other variables}

Clinical parameters included self-reported viral suppression, self-rated health (overall, physical, mental, and sexual), experience of ART side effects, comorbidities, concomitant medications, treatment satisfaction, and reasons for missing ART within the past month.
Demographic characteristics included age, gender, sexual orientation, domicile, and employment status. Poor selfprognosis regarding HIV mortality was an affirmative response ("Agree” or "Strongly agree”) to either statement below: "HIV will reduce my life span" or "Because of my HIV, I do not plan for my old age”.

\section{Analyses}

We performed the following: (1) computed the percentages of PLHIV with various treatment-related challenges and what percentage overall reported missing ART $\geq 1$ time in the past month due to the specified challenges. In line with the socio-ecological model's multi-layered approach to health issues [30], we examined challenges at the individual level (e.g., medical problems), interpersonal (e.g., perceived person-to-person stigma), and community level (e.g., perceived societal stigma). (2) Evaluated whether PLHIV with specific challenges/concerns were more likely to discuss those specific challenges/concerns with their HCP; comparisons were done using $\chi^{2}$ tests. (3) Analyzed the relationship between health-related outcomes and the extent of HCP-PLHIV engagement in care (low, moderate, high).

To examine co-occurrence of various treatment challenges, we tallied how many of the following treatment challenges were reported by each participant: ART side effects, difficulty swallowing, perceived stress from daily oral dosing, privacy/confidentiality concerns (hiding medications), and suboptimal adherence. These indicators were selected because they cut across the various domains of treatment challenges previously identified [24]. We created a dichotomous indicator for whether the participant reported $\geq 2$ (multiple) or $\leq 1$ (single or none) concurrent challenges to treatment. We examined the crude and adjusted relationships between number of treatment challenges reported and health-related outcomes such as self-rated health, perceived gaps with HIV treatment, perceived impact of HIV on their life, and sentiments of reduced life span because of HIV. The adjusted relationships were examined in a binary logistic regression model controlling for age and gender. Statistical significance was set at $\mathrm{p}<0.05$. All data analyses were performed with R Version 3.6.1.

\section{Results}

\section{Characteristics of study population}

A total of 60 PLHIV participated in the study; mean age was 41 years. The majority were men (78\% [47/60]), aged $<50$ years $(73 \%$ [44/60]) and reported $\geq 1$ non-HIV comorbidity (70\% [42/60]). Conditions with $>10 \%$ prevalence were: high cholesterol (27\% [16/60]), liver disease (22\% [13/60]), anemia (18\% [11/60]), mental illness $(18 \%$ [11/60]), gastrointestinal disease $(17 \%$ [10/60]), substance misuse (17\% [10/60]), insomnia (15\% [9/60]), heart disease $(13 \%[8 / 60])$, lung disease $(13 \%$ [8/60]), and lipodystrophy (12\% [7/60]). Prevalence of self-rated optimal health was as follows, by domain: 
+ Uి

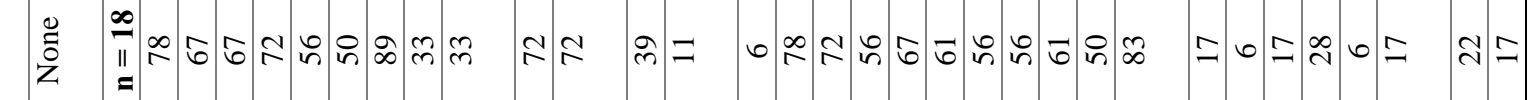

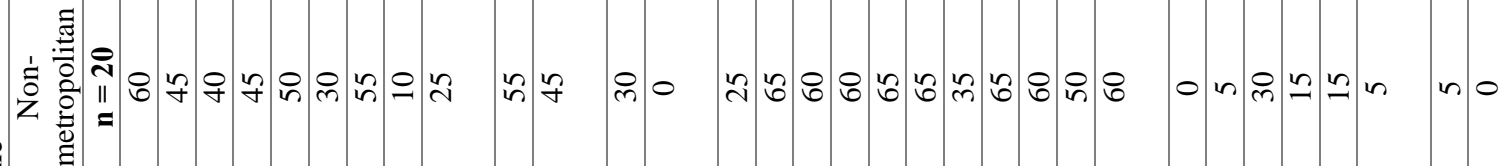
8 产

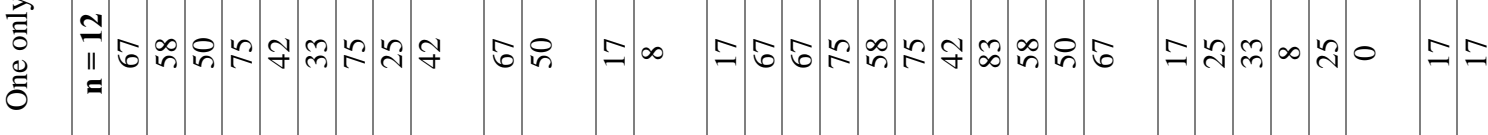

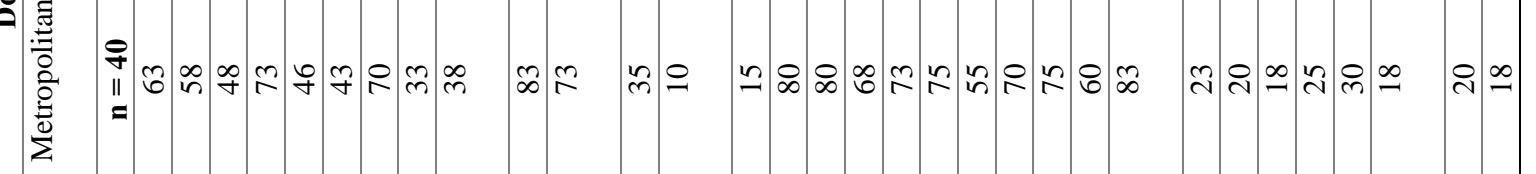

望

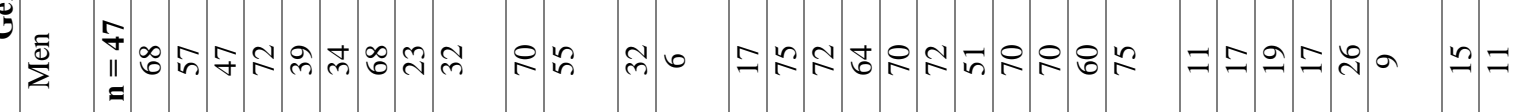

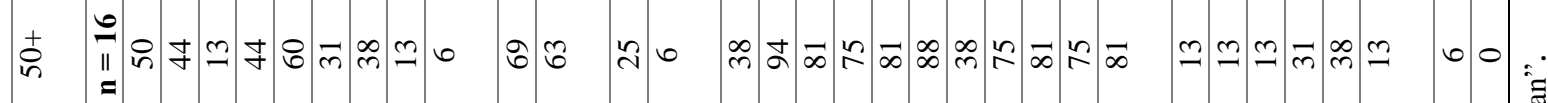
蕰

员 II

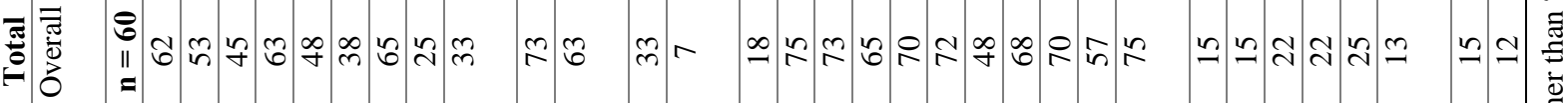

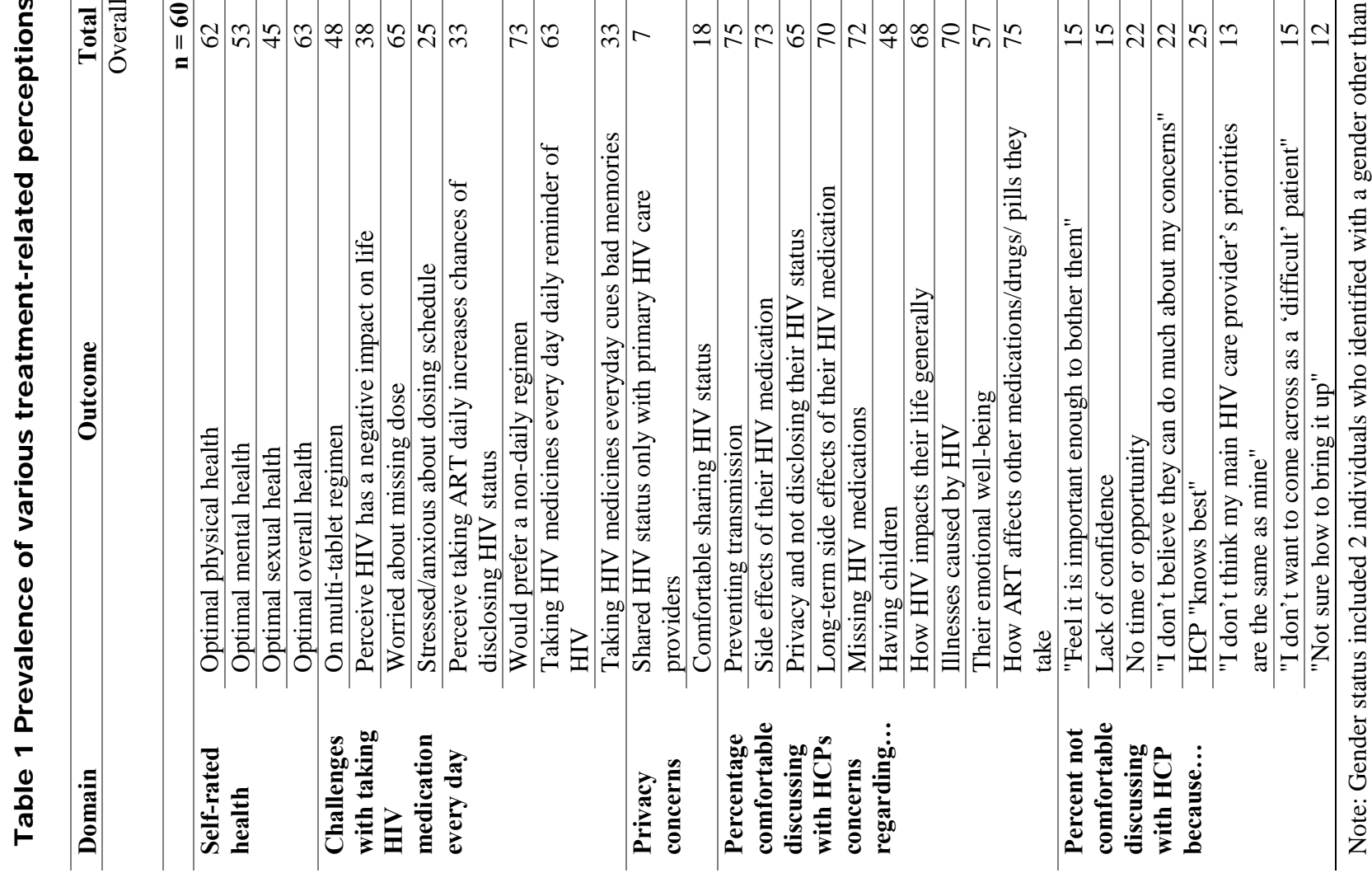


Figure 1 Individual, inter-personal, and community-level factors influencing HIV care $(N=60)$

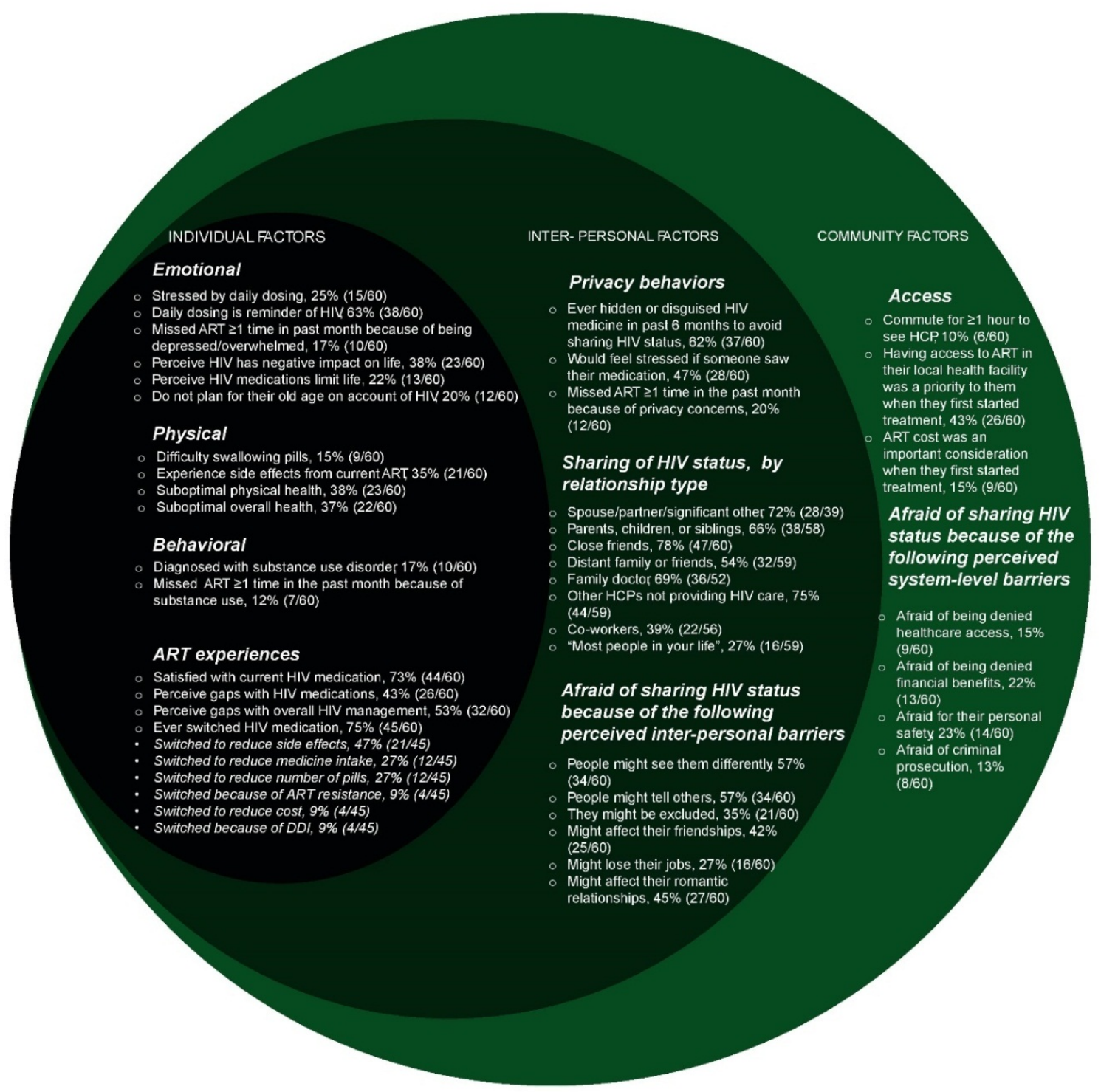

optimal physical health (62\% [37/60]), optimal mental health (53\% [32/60]), optimal sexual health (45\% [27/60]) and optimal overall health (63\% 38/60]) (Table 1).

\section{Treatment priorities, concerns, and adherence behaviors}

Respondents considered the following treatment goals to be most important to them when they first started their HIV treatment: (1) ensuring the virus was suppressed to prevent transmission to a partner (65\% [39/60]); (2) minimizing side effects (65\% [39/60]); (3) minimizing long-term negative impacts of treatment (60\% [36/60]); (4) flexible dosing schedule (e.g., with food, 60\% [36/60]); (5) managing symptoms or illnesses caused by HIV (52\% [31/60]); (6) keeping the number of medicines at a minimum (42\% [25/60]); (7) ensuring availability of the medicines in their public health facility (43\% [26/60]); (8) ensuring compatibility with concomitant medications (37\% [22/60]); (9) ensuring medication costs were affordable (15\% [9/60]) and (10) having children (15\% [9/60]).

Of all participants, 75\% [45/60] had ever switched their ART for any reason. Side effects were the most common reason for switching ART among those who ever switched (47\%, 21/45, Figure 1). Overall, 73\% [44/60] reported satisfaction with their current HIV medication, but $43 \%$ [26/60] felt there was room for improvement with their medication, and many were worried about running out of treatment options (58\% [35/60]). The vast majority (88\% [53/60]) were optimistic that future advances in HIV care would improve their overall wellbeing; of these, $85 \%$ [45/53] were open to taking ART with fewer medicines 
while 72\% [38/53] were open to long-acting ART, as long as they remained virologically controlled.

Even though almost the entire population reported being virally suppressed (97\% [58/60]), many experienced various challenges with treatment (Figure 1).

PLHIV reported a variety of physical, emotional, and psychosocial challenges with taking their ART. Physical challenges included difficulty ingesting medication (15\% [9/60]) and experience of ART side effects (35\% [21/60]). The majority of PLHIV expressed concerns about how taking ART for many years might impact their body and/or body shape (93\% [56/60]). Other ART-related concerns included anxiety over possibly having to take more medicines with increasing age (73\% [44/60]); concerns regarding risks of drug-drug interaction with ART $(63 \%$ [38/60]); or being worried about potentially unknown longterm impacts of ART (85\% [51/60]). Overall, 53\% [32/60] felt there was room for improvement with their overall HIV management.

ART-related privacy concerns constituted a major psychological challenge for most PLHIV, affecting HIV treatment adherence for some. Within the past 6 months, $62 \%$ [37/60] had ever hidden/disguised their HIV medication to prevent others from knowing they had HIV (Figure 2). More so, 47\% [28/60] indicated they would be stressed/anxious if someone were to see their HIV medication, of these $89 \%$ [25/28] were open to taking nondaily ARTs. Furthermore, 33\% [20/60] of all participants expressed concern that taking HIV medication everyday increased the chances of other people knowing they had HIV, of these 95\% (19/20) were open to taking non-daily ARTs. These privacy concerns were further reflected in the limited sharing of HIV status, especially with acquaintances such as co-workers among those with at least one co-worker (39\% [22/56]). Overall, 28\% [11/39] reported not having shared their HIV status even with a spouse/partner/significant other, among those with one. Notably, 15\% [9/60] reported not sharing their HIV status at least once in the past for fear they would be denied access to healthcare services. A similar percentage (13\% [8/60]) were afraid of sharing their HIV status for fear of prosecution.

Regarding emotional challenges related to daily ART, $22 \%$ [13/60] felt it limited their day-to-day life while $25 \%$ [15/60] were stressed by their daily dosing schedule. Furthermore, 63\% [38/60] said taking HIV medicines every day reminded them of their HIV status, 82\% [31/38] of these reported their preference for non-daily ARTs. More so, 33\% [20/60] of all participants associated daily oral dosing with bad memories, and 65\% [39/60] were worried about forgetting to take their ART as prescribed.

These challenges were associated with treatmentavoidance behaviors. For example, PLHIV reported missing ART $\geq 1$ time within the past month because of ART food requirements (27\% [16/60]), side effects (12\% [7/60]), concerns about long-term ART side effects $(10 \%$ $[6 / 60])$, and problems swallowing (5\% [3/60]). Furthermore, 20\% [12/60] missed ART $\geq 1$ time within the past month because they were in a situation they felt did not afford them enough privacy, while 17\% [10/60] missed $\geq 1$ time in the past month because they were depressed/overwhelmed.

Many PLHIV reported $\geq 2$ co-occurring treatment challenges. Of the five treatment challenges analyzed for co-occurrence (ART side effects, difficulty swallowing pills, perceived stress from daily oral dosing, hiding medications because of privacy concerns, and suboptimal adherence to treatment), only $17 \%$ [10/60] of the study population reported none of these challenges; 43\% [26/60] reported only one; $22 \%$ [13/60] reported only two, while $18 \%[11 / 60]$ reported $3+$ of the assessed treatment challenges. Of the 26 individuals reporting having only one of the assessed treatment challenge, 15/26 (58\%) reported only privacy concerns (hiding/disguising HIV medication), 7/26 (27\%) reported only side effects, 3/26 (11\%) reported only difficulty swallowing pills, whereas only 1/26 (4\%) reported solely suboptimal adherence. Of the 13 individuals who reported two treatment challenges only, the most common combinations were hiding/disguising medication plus stress over daily dosing schedule (5/13, $38 \%$ ), as well as hiding/disguising medication plus ART side effects $(5 / 13,38 \%)$.

Individuals with $\geq 2$ co-occurring treatment challenges reported significantly poorer health outcomes than those with $\leq 1$ treatment challenge, respectively, including perception HIV has a negative impact on their life (58\% [14/24] vs. 25\% [9/36], $\mathrm{p}=0.009$ ), and poor self-prognosis for HIV mortality (50\% [12/24] vs. 19\% [7/36], $\mathrm{p}=0.013)$. They were also more likely to perceive there was room for improvement with their HIV management (71\% [17/24] vs. $42 \%$ [15/36], $\mathrm{p}=0.027)$. After adjusting for age and gender, those with $\geq 2$ treatment challenges reported higher odds than those with $\leq 1$ treatment challenge, for the following: perceive HIV has a negative impact on their life (AOR $=3.94)$, perceive there is room for improving their overall HIV management (AOR $=4.65)$, perceive there is room for improving their HIV medication (AOR = 4.99), suboptimal overall health (AOR $=5.19)$, and poor selfprognosis for HIV mortality $(\mathrm{AOR}=10.90)($ all $\mathrm{p}<0.05)$.

\section{PLHIV-HCP engagement and the association with health outcomes}

Figure 3 highlights various PLHIV-reported indicators of engagement in care for both HCPs and PLHIV. For the HCP indicators, the lowest-performing one was a report by PLHIV that their HCP informed them of new treatment options (52\% [31/60]) whereas the highest HCP indicator was informing patients about Undetectable = Untransmittable (78\% [47/60]). The percentage of PLHIV reporting they were satisfied with their medication was significantly higher among PLHIV reporting vs. not reporting that their HCP: sought their views before prescribing treatment (84\% [32/38] vs. 55\% [12/22], p = $0.028)$; asked about their medication concerns $(83 \%$ [33/40] vs 55\% [11/20], $p=0.049$ ), and discussed newly available treatment options with them (87\% [27/31] vs. $59 \%$ [17/29], $\mathrm{p}=0.028)$. 
Figure 2. Percentage of participants who reported hiding their HIV medications, by selected demographic and clinical characteristics $(\mathrm{N}=60)$

\begin{tabular}{|c|c|c|}
\hline Total & Overall & $62 \%$ \\
\hline \multirow{2}{*}{ Age } & $<50$ y & $77 \%$ \\
\hline & $50+y$ & $19 \%$ \\
\hline \multirow[t]{3}{*}{ Comorbidity } & None besides HIV & $83 \%$ \\
\hline & One only & $75 \%$ \\
\hline & Two or more & $43 \%$ \\
\hline \multirow[t]{3}{*}{ Duration of HIV } & 2017 to 2019 & $80 \%$ \\
\hline & 2010 to 2016 & $75 \%$ \\
\hline & Pre-2010 & $42 \%$ \\
\hline \multirow[t]{2}{*}{ Location of PLHIV } & Metropolitan & $73 \%$ \\
\hline & Non-Metropolitan & $40 \%$ \\
\hline \multirow[t]{3}{*}{ Commute time to $\mathrm{HCP}$} & Under 30 minutes & $38 \%$ \\
\hline & 30 to 59 minutes & $80 \%$ \\
\hline & 1 hour or longer & $67 \%$ \\
\hline \multirow[t]{3}{*}{ Housing type } & Rent & $81 \%$ \\
\hline & Own & $33 \%$ \\
\hline & Other housing & $50 \%$ \\
\hline \multirow[t]{3}{*}{ Comedications } & None besides ART & $73 \%$ \\
\hline & Receiving medication for only one non-HIV condition & $69 \%$ \\
\hline & Receiving medication for two+ non-HIV conditions & $29 \%$ \\
\hline
\end{tabular}

Note: Chi-square tests for overall group differences were statistically significant for all indicators shown above at $\mathrm{p}<0.05$.

Figure 3 Percentage of participants who reported various indicators of engagement in care with their providers $(\mathrm{N}=60)$

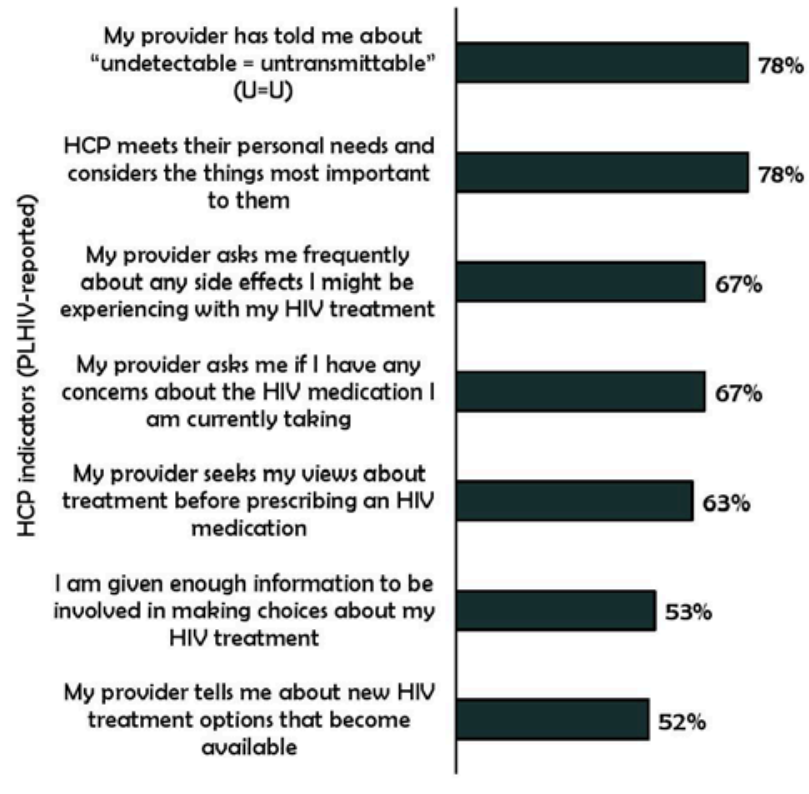

Percentage, \%

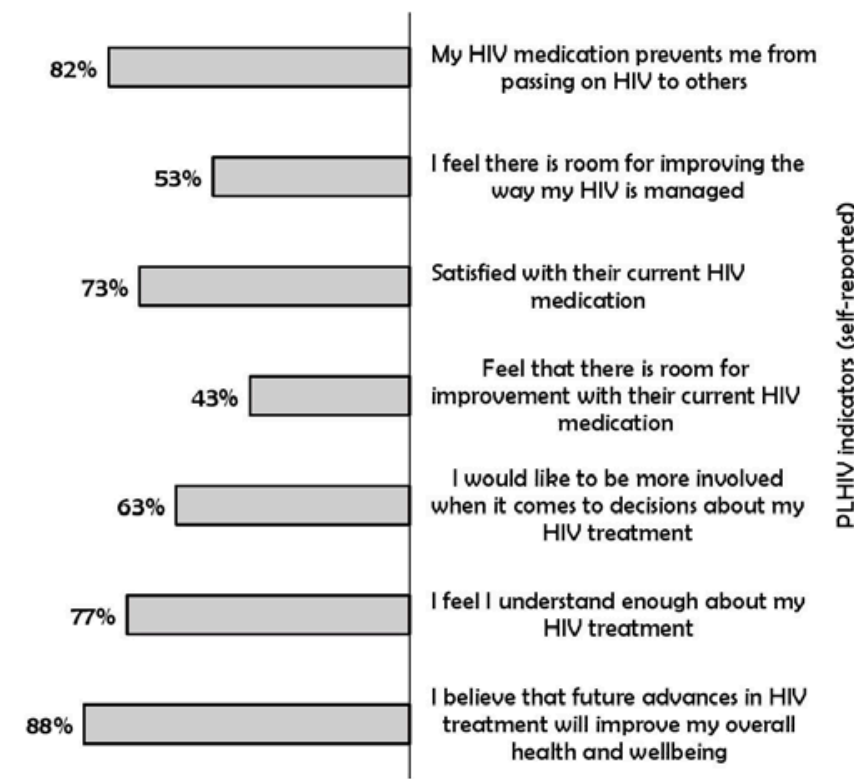

Percentage, \%
The indicators for PLHIV's own engagement showed widely varying levels of empowerment and self-efficacy towards their treatment. For example, while 82\% [49/60] indicated having knowledge of the number of medicines in their current HIV regimen, fewer indicated they understood enough about their treatment (77\% [46/60]). 


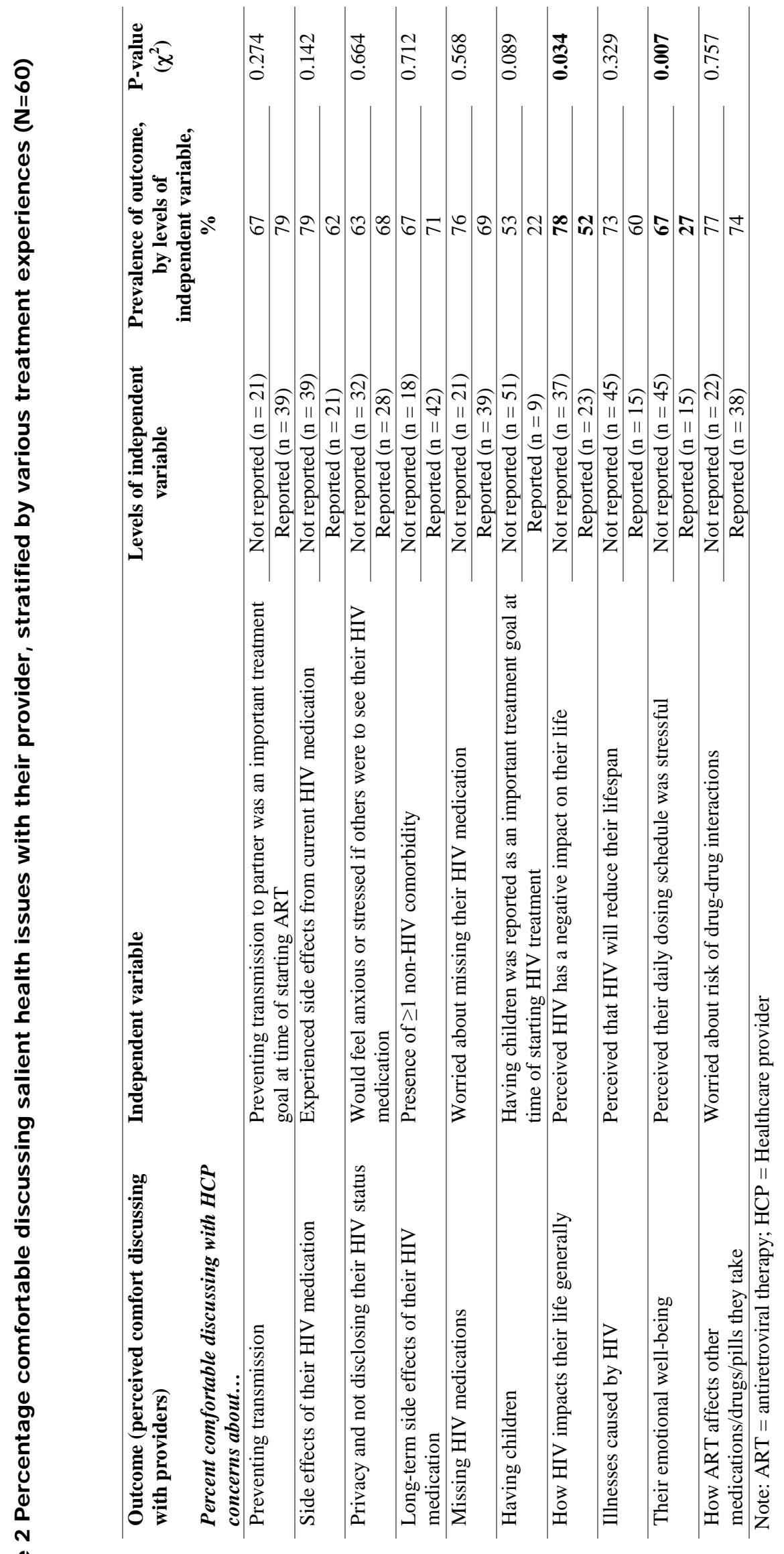




\section{Figure 4 Percentage of participants reporting various treatment-related perceptions, by extent of engagement in care $(\mathrm{N}=60)$. Note: $\mathrm{Chi}$-square tests for overall group differences were statistically significant for all indicators at $p<0.05$}

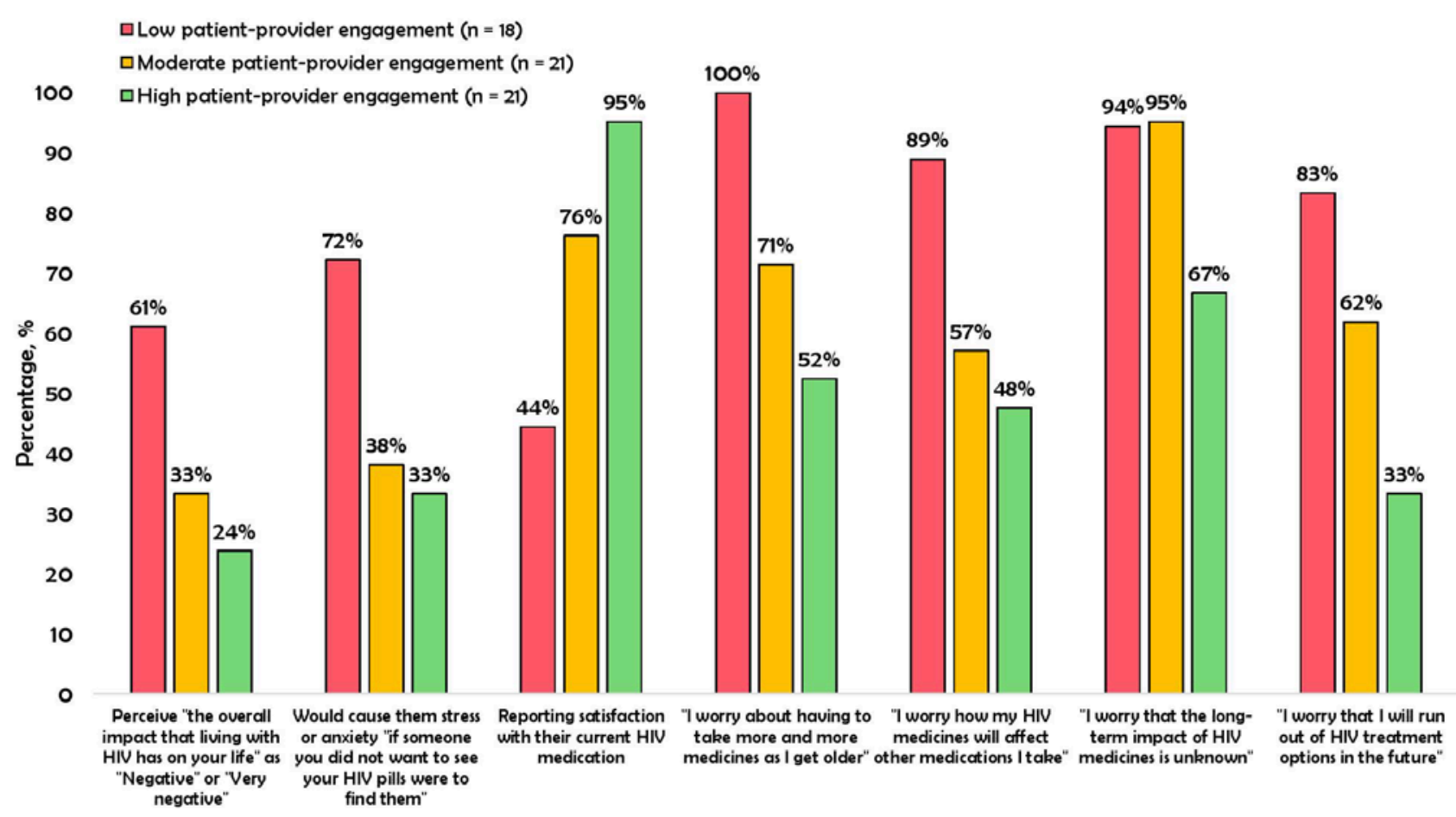

Similarly, 63\% [38/60] wanted to be more involved in making decisions about their HIV treatment, yet, a substantial proportion of these wanting more involvement were not comfortable discussing with their HCP key issues related to their care, including concerns about managing HIV symptoms or illnesses (37\%, 14/38), side effects (26\%, 10/38), and ART drug-drug interactions (24\%, 9/38). Furthermore, 43\% [26/60] felt there was room for improving their HIV medication, yet, close to half (46\% [12/26]) of these had never identified another medication they liked different from the regimen they were on, $19 \%$ [5/26]) ever identified one but never discussed it with their HCP; another 19\% [5/26] discussed with their HCP but were not prescribed; while $15 \%$ [4/26] received the prescription on asking.

Within the total sample, PLHIV reported varying levels of comfort with discussing treatment concerns with their HCPs, including concerns about preventing transmission (75\% [45/60]), drug-drug interactions (75\% [45/60]), ART side effects (73\% [44/60]), concerns about missing ART doses (72\% [43/60]), illnesses caused by HIV (70\% [42/60]), long-term ART side effects (70\% [42/60]), how HIV impacts their life generally (68\% [41/60]), privacy and not sharing their HIV status (65\% [39/60]), their emotional wellbeing (57\% [34/60]) and having children (48\% [29/60]).

We found a dissonance between experience of certain treatment challenges, and willingness to discuss those issues with HCPs (Table 2). For example, despite indicating that HIV had a negative impact on their day-today lives, only 52\% [12/23] of those reporting a negative impact from HIV (vs. 78\% [29/37] of those not reporting a negative impact, $\mathrm{p}=0.034$ ), were comfortable discussing how HIV impacts their life. Similarly, only 27\% [4/15] of those who found their daily dosing schedule stressful were comfortable discussing their emotional challenges with their HCP, compared to 67\% [30/45] of those not stressed by their daily dosing schedule $(\mathrm{p}=0.007)$. Furthermore, only $35 \%$ [21/60] of the entire sample felt they had no barriers to discussing any given issue with their HCPs; these individuals had more favorable health outcomes than those with a perceived communication barrier with their HCP, including higher prevalence of optimal physical (86\% [18/21] vs. 49\% [19/39], $\mathrm{p}=0.011)$ and mental health (86\% [18/21] vs. 36\% [14/39], p < 0.001). They also had significantly lower prevalence for the following negative outcomes: poor outlook for HIV mortality (5\% [1/21] vs. 46\% [18/39], $p=0.003$ ); perception HIV negatively impacts life $(14 \%$ [3/21] vs. $51 \%$ [20/39], $p=$ 0.011 ), and concerns regarding taking more and more medicines with age $(52 \%$ [11/21] vs. $85 \%$ [33/39], $\mathrm{p}=$ 0.017).

Overall, greater HCP engagement in care was associated with lower likelihood of reporting emotional and psychological challenges to treatment (Figure 4). PLHIV with the lowest engagement had almost three-fold higher prevalence of perceiving a negative impact from HIV (61\% [11/18] vs. 24\% [5/21], p = 0.049) and reporting they would be stressed or anxious if someone were to see their HIV medication (72\% [13/18] vs. 33\% [7/21], $\mathrm{p}=0.033$ ). Other results are shown in Figure 4. 


\section{Disc ussion}

PLHIV need and have perceived priorities that are diverse in our study, consistent with those reported in previous research [12,15,24]. Many PLHIV wanted improvements with their HIV medications (43\%), but even more wanted improvements with their overall HIV management (53\%). Physical, psychosocial, and emotional challenges, including anticipated stigma, were common among PLHIV, as noted in previous research [2,31]. Some of these treatment concerns were associated with treatment avoidance behaviors, and PLHIV with multiple, cooccurring treatment challenges had worse outcomes than those with only one or none. PLHIV reported to different levels, preferences for innovative treatments, namely the reduction of the number of medicines in their treatment and/or treatments requiring less frequent intakes, as long as their viral suppression is maintained.

Given evidence of a strong relationship between adherence to daily oral ART and treatment satisfaction [15,32-34], providing flexible treatment options to patients may increase treatment adherence and retention in care. New non-oral long-acting directly observed ARTs could potentially address some of the unmet problems identified in this study. Long acting ART and recipients' resulting attitudes about the therapy were evaluated qualitatively as part of the phase IIb trial LATTE-2 [35,36]; interviewed patients were willing to tolerate adverse events associated with long-acting injections, in part because of the greater convenience and the ease of incorporating into routines. PLHIV who reported that their HCP sought their views before prescribing treatment were more likely to report treatment satisfaction, underscoring the need for HCPs to incorporate patients' treatment preferences when starting or switching ART [25].

Despite being a relatively young population (mean age of 41 years), $70 \%$ reported a non-HIV comorbidity, and most were concerned about the short, intermediate, or long-term effects of HIV medicines, such as the potential for drug-drug interactions, side effects - both known and unknown, as well as anxiety over a lifetime of medication intake. Preference for non-daily ARTs among PLHIV is not solely driven by considerations of potential pathophysiological effects of medicines. On the contrary, there are also psychological factors at play [23]. For example, the daily effect of oral ART intake as a reminder of HIV status, and as a cause for self-discrimination and its cascade is a significant barrier to daily oral dosing; 95\% of PLHIV concerned that daily dosing increases likelihood of others knowing their HIV status were open to taking nondaily ARTs. Similarly, $89 \%$ of PLHIV that would be anxious or stressed if someone were to see their HIV medication were open to taking non-daily ARTs.

In the 2020 Global AIDS update, Portugal is acknowledged as one of the countries to have "nearly achieved 2020 target (85-89\%)" in relation to the second, and third targets of the Joint United Nations Programme on HIV/AIDS [1,37]. Much work remains to be done to make significant progress towards the fourth "90" (improved quality of life) among PLHIV in Portugal [16].
Acknowledging the intersection between HIV and emotional, mental, and physical aspects of life, including multimorbidity and polypharmacy, can inform holistic care. To meet the fourth "90" target of improving quality of life among PLHIV, enhanced and sustained efforts are needed to implement holistic care that considers patients' concerns, co-morbidities, priorities, and preferences when starting or switching HIV medication to minimize the impact of HIV treatment on daily life.

Unfortunately, some who needed help the most were the least likely to seek it out from their HCP; for example, only $27 \%$ of those stressed by their daily dosing schedule were comfortable discussing their emotional challenges with their HCP, compared to $67 \%$ among those not stressed by their medication. An alternative explanation for this is however reverse causation; it may well be that the current perception of not feeling stressed was preceded by an HCP intervention. Taken together with our finding that those who reported barriers in communicating with their HCP had more unfavorable health outcomes, these findings suggest a benefit from more meaningful engagement between PLHIV and their HCPs. We also observed a strong gradient, where high HCP-PLHIV engagement was associated with more favorable health outcomes of several-fold magnitude that those with low engagement. Several of our outcomes were current attitudes and perceptions at the time of the survey; this mitigates temporality biases as the exposure variable (engagement with HCPs) occurred in the past and preceded the outcomes of interest. Our findings underscore the need for barrier-free, quality communication between patients and their providers to facilitate sharing of salient health information and improve treatment outcomes as well as quality of life.

Our study's strength is recruitment of individuals with confirmed HIV status, ensuring a well-defined study population. The data are recent and therefore relevant to the current landscape. Nonetheless, this study also has some limitations. We had a limited sample size which may have resulted in less precise estimates. The cross-sectional survey is subject to temporality biases and only associations can be drawn. The self-reports may be subject to misreporting and misclassification bias. Finally, the study may have limited generalizability because of the non-probabilistic sampling and use of web-based surveys.

\section{Conclusion}

Physical, emotional, and psychosocial challenges were common among PLHIV and were associated with treatment avoidance behaviors. Individuals with multiple treatment challenges occurring simultaneously had worse health-related outcomes. For some PLHIV, taking pills daily was linked with diverse emotional challenges, including pill fatigue and anxiety. PLHIV wanted improvements in their HIV medications and in their overall HIV management, reporting preference for HIV treatments with less medications and/or non-daily dosing. HCPs should encourage PLHIV to voice their concerns, since 
many patients who need help may be hesitant to ask for it. Patient involvement in treatment decisions had a significant association with positive health outcomes, including treatment satisfaction. HCPs should inform patients of new treatment options and involve them when starting and switching treatments. Clinicians should consider patient preferences when prescribing ART to enhance their health-related quality of life.

\section{Acknowledgements and Conflicts of Interest}

Data analyses and medical writing services were supported by Zatum LLC. The authors are employees of ViiV Healthcare. No copyrighted materials, surveys, instruments, or tools were used in this study. Financial support for primary data collection came from ViiV Healthcare. Major thematic areas explored within the survey were developed in consultation with people living with HIV, patient organizations and HIV healthcare providers to ensure that issues of importance to people living with HIV were adequately captured.

\section{References}

[1] Brown, A.E., Hayes, R., Noori, T., Azad, Y., AmatoGauci, A.J., Pharris, A., Delpech, V.C. \& The ECDC Dublin Declaration Monitoring Network (2018). HIV in Europe and Central Asia: progress in 2018 towards meeting the UNAIDS 90-90-90 targets. Euro Surveillance 23 (48) 1800622.

[2] Bravo, P., Edwards, A., Rollnick, S. \& Elwyn, G (2010). Tough decisions faced by people living with HIV: a literature review of psychosocial problems. AIDS Reviews 12 (2) 76-88.

[3] Antori, A., Arendt, G., Becker, J.T. et al. (2007). Updated research nosology for HIV-1 associated neurocognitive disorders. Neurology 69 (18) 1789-1799.

[4] Tross, S., Price, R.W., Navia, B., Thaler, H.T., Gold, J., Hirsch, D.A. \& Sidtis, J.J. (1988). Neuropsychological characterization of the AIDS dementia complex: a preliminary report. AIDS 2 (2) 81-88.

[5] Tan, I.L., Smith, B.R., von Geldern, G., Mateen, F.J. \& McArthur, J.C. (2012). HIV-associated opportunistic infections of the CNS. The Lancet Neurology 11 (7) 605617.

[6] Guimarães, M.D., McKinnon, K., Campos, L.N., Melo, A.P. \& Wainberg, M. (2010). HIV risk behavior of psychiatric patients with mental illness: A sample of Brazilian patients. Revista Brasileira de Psiquiatria 32, 349-360.

[7] Meade, C.S. \& Sikkema, K.J. (2005). HIV risk behavior among adults with severe mental illness: A systematic review. Clinical Psychology Review 25, 433457.

[8] Manji, H., Jäger, H. \& Winston, A. (2013). HIV, dementia and antiretroviral drugs: 30 years of an epidemic.
Journal of Neurology, Neurosurgery \& Psychiatry 84 (10) 1126-1137.

[9] HIV Justice Network and the Global Network of People Living with HIV (GNP+). Advancing HIV Justice 2: Building momentum in global advocacy against HIV criminalisation. Available at

https://www.scribd.com/doc/312008825/Advancing-HIVJustice-2-Building-momentum-in-global-advocacyagainst-HIV-criminalisation._(last accessed March 20, 2020).

[10] Smith, R., Rossetto, K. \& Peterson, B.L. (2008). A meta-analysis of disclosure of one's HIV-positive status, stigma and social support. AIDS Care 20, 1266-1275.

[11] Stutterheim, S.E., Pryor, J.B., Bos, A.E., Hoogendijk, R., Muris, P. \& Schaalma, H.P. (2009). HIV-related stigma and psychological distress: The harmful effects of specific stigma manifestations in various social settings. AIDS 23, 2353-2357.

[12] Okoli, C., de Los Rios, P., Eremin, A., Brough, G., Young, B. \& Short, D. (2020). Relationship Between Polypharmacy and Quality of Life Among People in 24 Countries Living With HIV. Preventing Chronic Disease 17, E22.

[13] Altice, F., Evuarherhe, O., Shina, S., Carter, G. \& Beaubrun, A.C. (2019). Adherence to HIV treatment regimens: systematic literature review and meta-analysis. Patient Preference and Adherence 13, 475-490.

[14] Basavaraj, K.H., Navya, M.A. \& Rashmi, R. (2010). Quality of life in HIV/AIDS. Indian Journal of Sexual Transmitted Diseases and AIDS 31 (2) 75-80.

[15] de Los Rios, P., Okoli, C., Punekar, Y. et al. (2020). Prevalence, determinants, and impact of suboptimal adherence to HIV medication in 25 countries. Preventive Medicine 139, 106182.

[16] Lazarus, J.V., Safreed-Harmon, K., Barton, S.E. et al. (2016). Beyond viral suppression of HIV - the new quality of life frontier. BMC Medicine 14 (1) 94.

[17] UNAIDS Data 2018. Available at: https://www.aidsdatahub.org/unaids-data-2018-unaids2018. (last accessed March 20, 2020).

[18] World Health Organization. Constitution. Available at: https://www.who.int/about/who-we-are/constitution. (last accessed September 19, 2019).

[19] Buetow S.A. (1995). What do general practitioners and their patients want from general practice and are they receiving it? A framework. Social Science \& Medicine 40 (2) 213-221.

[20] Schäfer, W., Boerma, W., Schellevis, F.G. \& Groenewegen, P.P. (2018). GP Practices as a One-Stop Shop: How Do Patients Perceive the Quality of Care? A Cross-Sectional Study in Thirty-Four Countries. Health Services Research 53 (4) 2047-2063.

[21] Husain-Gambles, M., Neal, R.D., Dempsey, O., Lawlor, D.A. \& Hodgson, J. (2004). Missed appointments in primary care: questionnaire and focus group study of health professionals. British Journal of General Practice 54 (499) 108-113.

[22] October, T.W., Hinds, P.S., Wang, J., Dizon, Z.B., Cheng, Y.I. \& Roter, D.L. (2016). Parent Satisfaction With Communication Is Associated With Physician's PatientCentered Communication Patterns During Family 
Conferences. Pediatric Critical Care Medicine 17 (6) 490497.

[23] de Los Rios, P., Okoli, C., Young, B. et al. (2020).

Treatment aspirations and attitudes towards innovative medications among people living with HIV in 25 countries. Population Medicine Available at: https://doi.org/10.18332/popmed/124781.

[24] de Los Rios, P., Okoli, C., Castellanos, E. et al. (2020). Physical, emotional, and psychosocial challenges associated with daily dosing of HIV medications and their impact on indicators of quality of life: Findings from the Positive Perspectives Study. AIDS and Behavior In press.

[25] Okoli, C., Brough, G., Allan, B., et al. (2020). Shared Decision Making Between Patients and Healthcare Providers and its Association with Favorable Health Outcomes Among People Living with HIV. AIDS and Behavior 1-12. Advance online publication. Available at: https://doi.org/10.1007/s10461-020-02973-4.

[26] Delivering quality health services: a global imperative for universal health coverage. Geneva: World Health Organization, Organisation for Economic Co-operation and Development, and The World Bank; 2018. License: CC BY-NC-SA 3.0 IGO. Available at: http://documents.banquemondiale.org/curated/fr/48277153 0290792652/pdf/127816-REVISED-quality-jointpublication-July2018-Complete-vignettes-ebook-L.pdf. (last accessed March 20, 2020).

[27] Healthcare Personnel Statistics - Physicians. Statistics Explained. Available at:

https://ec.europa.eu/eurostat/statisticsexplained/. (last accessed November 29, 2019).

[28] World Health Organization Regional Office for

Europe. HIV/AIDS surveillance in Europe 2019. Available at: https://www.ecdc.europa.eu/en/publications-

data/hivaids-surveillance-europe-2019-2018-data.

Accessed 08/09/2020.

[29] Elwyn, G., Edwards, A., Wensing, M., Hood, K., Atwell, C. \& Grol, R. (2003). Shared decision making: developing the OPTION scale for measuring patient involvement. Quality and Safety in Health Care 12 (2) 9399.

[30] McLeroy, K.R., Bibeau, D., Steckler, A. \& Glanz, K. (1988). An Ecological Perspective on Health Promotion Programs. Health Education Quarterly 15, 351-377.

[31] Okoli, C., Van de Velde, N., Richman, B. et al. (2020). Undetectable equals untransmittable $(U=U)$ : awareness and associations with health outcomes among people living with HIV in 25 countries. Sexually Transmitted Infections Available at: https://doi.org/10.1136/sextrans-2020-054551.

[32] Jordan, J., Cahn, P., Goebel, F., Matheron, S., Bradley, C. \& Woodcock, A. (2005). Abacavir compared to protease inhibitors as part of HAART regimens for treatment of HIV infection: patient satisfaction and implications for adherence. AIDS Patient Care and STDs 19 (1) 9-18.

[33] Ventura Cerda, J.M., Martin Conde, M.T., Morillo Verdugo, R., Tebenes Cortes, M. \& Casado Gomez, M.A (2014). Adherence, satisfaction and health-related quality of life in HIV-infected patients with antiretroviral therapy in Spain. The ARPAS study. Farmacia hospitalaria: organo oficial de expresion cientifica de la Sociedad Espanola de Farmacia Hospitalaria 38 (4) 291-299.

[34] Boretzki, J., Wolf, E., Wiese, C., Noe, S., Balogh, A., Meurer, A., Krznaric, I., Zink, A., Lersch, C. \& Spinner, C.D. (2017). Highly specific reasons for nonadherence to antiretroviral therapy: results from the German adherence study. Patient Preference and Adherence 11, 1897-1906.

[35] Kerrigan, D., Mantsios, A., Gorgolas, M. et al. (2018). Experiences with long acting injectable ART: a qualitative study among PLHIV participating in a Phase II study of cabotegravir + rilpivirine (LATTE-2) in the United States and Spain. PloS One 13 (1) e0190487.

[36] Murray, M., Dorey, D., Griffith, S., Mrus, J., Spreen, W. \& Margolis, D. (2016). Satisfaction, tolerability, and acceptability or cabotegravir (CAB) + rilpivirine (RPV) long-acting therapy: LATTE-2 results [abstract THPEB052]. Abstract presented at 21st International AIDS Conference (AIDS); July 18-22; Durban, South Africa. [37] Global AIDS Update (2020). Seizing The Moment. Tackling entrenched inequalities to end epidemics. Available at:

https://www.unaids.org/en/resources/documents/2020/glob al-aids-report. Accessed 08/09/2020. 